\title{
A Clustering Routing Algorithm for Heterogeneous Sensor Networks with Multilevel Energies
}

\author{
Huaixiang Zhang*, Qingcong Cao and Lu Yin \\ School of Computer Science and Technology, Hangzhou Dianzi University, Hangzhou, Zhejiang 310018, China \\ *Corresponding author
}

\begin{abstract}
In clustering algorithm of wireless sensor networks, to solve the problem of excessive energy consumption in the cluster heads with multilevel energies, an improved reactive three level heterogeneous stable election protocol is proposed by combining the reactive routing mechanism and multilevel heterogeneous networks. In cluster heads election phase of every round, the algorithm considers the residual energy and higher initial energy of all nodes to elect an optimal cluster head. Furthermore, reactive routing mechanism is employed here to minimize the number of nodes transmitting data to the cluster heads. Simulation results show that the improved routing protocol has better performance in the network life cycle and network energy consumption than SEP, SEP-E and ESEP-E protocol respectively such that it is more suitable for dealing with the problem of multilevel energy heterogeneous sensor network.
\end{abstract}

Keywords-heterogeneous sensor networks; cluster head election; reactive routing

\section{INTRODUCTION}

Wireless sensor networks (WSNs) is widely considered as one of the most important technologies for the twenty-first century [1]. Over the past nearly two decades, it has been aroused great attention, including academia, industry, military and other domain. A WSN typical consists of a large number of low-cost, low-power, and multifunctional sensor nodes equipped with embedded microprocessors, radio receivers, and power components to enable sensing, computing, communication, and actuation [2].In WSNs, the primary design goal is the effective use of energy, which becomes a hot issue in WSNs research.

A lot of routing protocols have been proposed to extend the network lifetime. According to the initial energy, different power, hardware of the sensor nodes, WSNs can be classified into heterogeneous wireless sensor networks and homogeneous wireless sensor networks [3]. In terms of saving the energy consumption of heterogeneous nodes, routing protocols can be divided into reactive routing protocol and proactive routing protocol [4]. For the proactive routing protocols, nodes continuously monitor surrounding environment and transmits sensor data at a constant rate. However, for the reactive routing protocols, only when changes happen in the sensor data can the nodes be active. Considered the network topology, the WSNs routing protocols can be categorized into flat routing and hierarchical routing [5].The flat routing protocol bears simple structure, but it's quite difficult to build and maintain, and thereby it's suitable for small scale. Contrarily, hierarchical routing protocol has more advantages in load balancing, scalability, and energy efficiency. The typical hierarchical routing protocols lie in LEACH [6], HEED [7], LEACH-C [8], etc.

In recent years, there are a wide range of researches on the routing protocol of heterogeneous WSNs. A two level heterogeneous Stable Election Protocol (SEP) consisting of two types of nodes according to the initial energy was provided [9]. The advanced nodes are equipped with more initial energy than the normal nodes and have a greater probability to become cluster head node. SEP protocol can get a longer life cycle in the two level heterogeneous WSNs, but it's still not good enough to be competent in multilevel heterogeneous network environments. Paper [10] declared a three level heterogeneous protocol called SEP-E, which is an improved protocol based on SEP and more reasonable use of the network energy. Even so, SEP-E can barely consume the normal energy to elect a cluster head when the environment changes. Meanwhile, an enhancement protocol named ESEP-E [11] was developed on the basis of SEP-E. Though ESEP-E is superior to SEP-E in the cluster head election by means of optimizing the probability, it still cannot maximize the network life cycle.

The paper proposes an improved reactive three level energy heterogeneous stable election protocol (RTL-HSEP), putting an emphasis on the analysis of the residual energy estimation and reactive routing mechanism to reduce the data transmission between nodes. RTL-HSEP fully exploits the remaining energy and higher initial energy to re-select the cluster heads, making it more viable to optimize cluster head electing and maximize the network life cycle.

\section{THE NETWORK MODEL}

In order to verify the performances of the proposed protocol, some assumptions for the heterogeneous network model are described as below:

(1) Nodes randomly deployed within a square monitoring area, with the location of the node unknown and the base station position fixed.

(2) WSN consists of heterogeneous nodes in terms of node energy and the base station has enough energy in comparison with other nodes in the network.

(3) Communication power for all nodes can be easily adjusted according to the transmission distance.

The radio propagation model adopted here is similar to the one described in SEP. The energy dissipation for a node transmitting $k$ bits data in a distance $d$, denoted as $E_{t x}$, 
can be defined as

$$
E_{t x}(k, d)=\left\{\begin{array}{l}
E_{\text {elec }} * k+\varepsilon_{f_{s}} * k^{*} d^{2}, d<d_{0} \\
E_{\text {elec }} * k+\varepsilon_{m p} * k^{*} d^{4}, d \geq d_{0}
\end{array} .\right.
$$

where $E_{\text {elec }}$ represents the energy dissipated per bit to run the transmitter or the receiver circuit, $d_{0}$ indicates a threshold. Additionally, $E_{f s}$ and $E_{m p}$ represent the energy required in free space and multipath fading environments respectively.

Similarly, the energy dissipation for a node receiving the $k$ bits data, identified as $E_{r x}$, satisfies

$$
E_{r x}(k)=k^{*} E_{\text {elec }}
$$

\section{The Proposed RTL-HSEP AlgorithM}

The proposed RTL-HSEP algorithm is established by combining the reactive routing mechanism and multilevel heterogeneous networks. It's composed of three different types of nodes with different level energies, which results in a difficulty to elect the proper cluster heads. Meanwhile, reactive routing mechanism is employed here to minimize the number of nodes transmitting data to the cluster heads so as to save the energy.

\section{A. Cluster Head Election with Multilevel Energies}

Among three-level heterogeneous WSNs, the nodes are categorized into advanced nodes, middle nodes and normal nodes. Here, the total number of nodes is identified as $n$ and the initial energy for each normal node as $E_{0}$.

Assume that advanced nodes and middle nodes both have more initial energy than the normal nodes. Let $m$ be the fraction of the total number of nodes $n$, which are equipped with $a$ times more energy than the normal nodes. We refer to these powerful nodes as advanced nodes, and $b$ is the proportion of middle nodes to the total number of nodes $n$ with $u$ times more energy than the normal nodes. The rest of $n(1-m-b)$ nodes are deployed as normal nodes with initial energy $E_{0}$. Consequently, the initial energy of advanced nodes is $E_{0}(1+a)$ and for middle nodes, $E_{0}(1+u)$. We assume $u=a / 2$.

The total energy of normal nodes, middle nodes and advance nodes can be expressed as $n . E_{0}(1-m-b), n . b . E_{0}(1+u), n . m . E_{0}(1+a)$ respectively. In order to make the entire network load balance, assume that advance nodes and middle nodes possess more chances than normal probability to be elected as cluster heads. Let $p_{\text {opt }}$ represent the optimal probability of a node to become a cluster head. The proposed algorithm should satisfy the following rules:
(1) In every $\frac{1}{p_{\text {opt }}}(1+m \cdot a+b \cdot u)$ round, each normal node is elected as a cluster head ;

(2) In every $\frac{1}{p_{\text {opt }}}(1+m \cdot a+b . u)$ round, each middle node is elected exactly $(1+u)$ times as a cluster head;

(3) In every $\frac{1}{p_{\text {opt }}}(1+m \cdot a+b \cdot u)$ round, each advance node is elected exactly $(1+a)$ times as a cluster head;

Therefore, the probabilities to be elected as a cluster head for normal nodes, middle nodes and advanced nodes, termed as $p_{\text {nrm }}, p_{\text {mid }}, p_{a d v}$ respectively, are defined as

$$
\begin{gathered}
p_{n r m}=\frac{p_{o p t}}{1+m^{*} \alpha+b^{*} u} * \frac{E_{i}(r)}{\bar{E}(r)} . \\
p_{\text {mid }}=\frac{p_{o p t}(1+u)}{1+m^{*} \alpha+b^{*} u} * \frac{E_{i}(r)}{\bar{E}(r)} . \\
p_{a d v}=\frac{p_{o p t}(1+\alpha)}{1+m^{*} \alpha+b^{*} u} * \frac{E_{i}(r)}{\bar{E}(r)} .
\end{gathered}
$$

where $k_{\text {opt }}$ represents the number of optimal cluster heads, $E_{i}(r)$ refers to the residual energy of node $i$ at round $r \cdot \bar{E}(r)$ denotes the average energy of the network at round $r$, given by

$$
\bar{E}(r)=\frac{1}{n} \sum_{i=1}^{n} E_{i}(r)
$$

In order to ensure that cluster head election is done in the same way as SEP, SEP-E and ESEP-E do, similar thresholds for normal nodes, middle nodes and advanced nodes, termed as $T_{n r m}, T_{\text {mid }}, T_{a d v}$ respectively, are introduced. For each type of nodes, a random number between 0 and 1 is generated and thereby used to compare with the corresponding threshold value. On the condition that the random number is less than the corresponding threshold value, the associated nodes will be elected as a cluster head and broadcast messages. The threshold for each type of nodes is proposed as

$$
T(n r m)=\left\{\begin{array}{ll}
\frac{p_{n r m}}{1-p_{n r m} *\left(r \bmod \frac{1}{p_{n r m}}\right)}, & n_{n r m} \in \mathrm{G}^{\prime} \\
0, & \text { otherwise }
\end{array} .\right.
$$




$$
\begin{gathered}
T(\text { mid })=\left\{\begin{array}{ll}
\frac{p_{\text {mid }}}{1-p_{\text {mid }} *\left(r \bmod \frac{1}{p_{\text {mid }}}\right)}, & n_{\text {mid }} \in \mathrm{G}^{\prime \prime} \\
0, & \text { otherwise }
\end{array} .\right. \\
T(a d v)= \begin{cases}\frac{p_{a d v}}{1-p_{a d v} *\left(r \bmod \frac{1}{p_{a d v}}\right)}, & n_{a d v} \in \mathrm{G}^{\prime \prime \prime} \\
0, & \text { otherwise }\end{cases}
\end{gathered}
$$

where $G^{\prime}, G^{\prime \prime}$ and $G^{\prime \prime \prime}$ represent the set of normal nodes, middle nodes and advanced nodes that has not been elected as cluster head until now, respectively.

As a result, the average number of elected cluster head per round follows

$$
n \cdot(1-m-b) \cdot p_{n r m}+n \cdot b \cdot p_{m i d}+n \cdot m \cdot p_{a d v}=n \cdot p_{o p t} \text {. }
$$

\section{B. Reactive Routing Mechanism}

Unlike the usual routing algorithm provided in SEP, ESEP, etc., the proposed RTL-HSEP employs a different mechanism in the data transfer phase. Besides broadcasting TDMA to allocate the time-slot for all cluster members, RTL-HSEP also takes the responsibility for broadcasting the soft and hard threshold values. Hard threshold refers to the value which detected data can't exceed, and soft threshold refers to the acceptable fluctuation range of detected data. These threshold values are established and maintained periodically by cluster heads.

Cluster members are continuously detecting the surrounding environments. Once the sensor data is close to the predefined hard threshold value, transmitter is turned on and the sensor data are transmitted to cluster head. Only when conditions that sensor data is bigger than the hard threshold value and the difference between the last sensor data is not less than the soft threshold value are both satisfied, can the node be able to transmit and receive sensor data again. Meanwhile, sensor data will certainly not be transmitted to its belonged cluster head when the difference between the last sensor data is smaller than the soft threshold value. This mechanism can effectively reduce the chance for sensor node trying transferring sensor data to cluster head.

\section{Simulation Results}

\section{A. Simulation Parameters}

To verify the performance of the RTL-HSEP protocol, we used MATLAB platform to implement the simulation. Simulation parameters are shown in Table 1. The network model has $n=150$ sensor nodes, which are deployed randomly in a $150 \times 150$ square meters region. Base station is located in the center of the sensing region. Set initial energy $E_{0}=0.5 \mathrm{~J}, a=2$.To reduce the complexity of the simulation, the radio channel interference is not taken into consideration. The performance of RTL-HSEP is compared with SEP, SEP-E, and ESEP-E in terms of lifetime of the network and data packets the base station received.

TABLE I. SIMULATION PARAMETERS

\begin{tabular}{|l|l|}
\hline Experimental parameters & value \\
\hline Network area & $150 \times 150\left[\mathrm{~m}^{2}\right]$ \\
\hline Number of nodes & 150 \\
\hline Location of base station & $(75,75)[\mathrm{m}]$ \\
\hline Packet size & $4000[\mathrm{bits}]$ \\
\hline$P_{\mathrm{opt}}$ & 0.1 \\
\hline$E_{0}$ & $0.5[\mathrm{~J}]$ \\
\hline$\varepsilon_{\mathrm{fs}}$ & $10\left[\mathrm{pJ} / \mathrm{bit} / \mathrm{m}^{2}\right]$ \\
\hline$\varepsilon_{\mathrm{mp}}$ & $0.0013\left[\mathrm{pJ} / \mathrm{bit} / \mathrm{m}^{4}\right]$ \\
\hline$E_{\mathrm{DA}}$ & \\
\hline$a$ & $5[\mathrm{~nJ} / \mathrm{bit} / \mathrm{message}]$ \\
\hline$m$ & 2 \\
\hline$E_{\text {elec }}$ & 0.2 \\
\hline$u$ & $50[\mathrm{~nJ} / \mathrm{bit}]$ \\
\hline$b$ & 1 \\
\hline & 0.2 \\
\hline
\end{tabular}

\section{B. Performance Evaluation}

Fig. 1 plots the number of dead nodes per round and they are much less in RTL-HSEP as compared to other protocols. The number of alive nodes per round is shown in Fig. 2 and from the graph it clearly shows the advantages of RTL-HSEP over the other protocols. As can be seen from Fig. 1 and 2, the stable period of RTL-HSEP protocol is extended greatly. It means that the network lifetime of RTL-HSEP is explicitly more than SEP, SEP-E and ESEP-E.

In both Fig. 1 and 2, ESEP-E performs better than SEP and SEP-E. But we can see that the unstable region of ESEP-E is larger than that of RTL-HSEP protocol. The reactive routing mechanism equipped in RTL-HSEP to reduce the number of nodes transmission accounts for it. It can be concluded that RTL-HSEP protocol is more suitable for heterogeneous networks and effectively extend the life cycle of wireless sensor network.

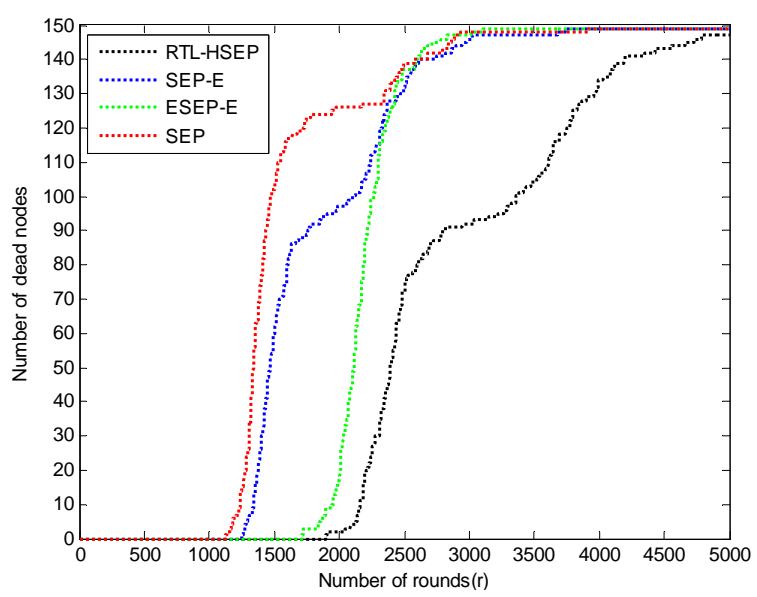

FIGURE I. NUMBER OF NODES DEATHS PER ROUND 


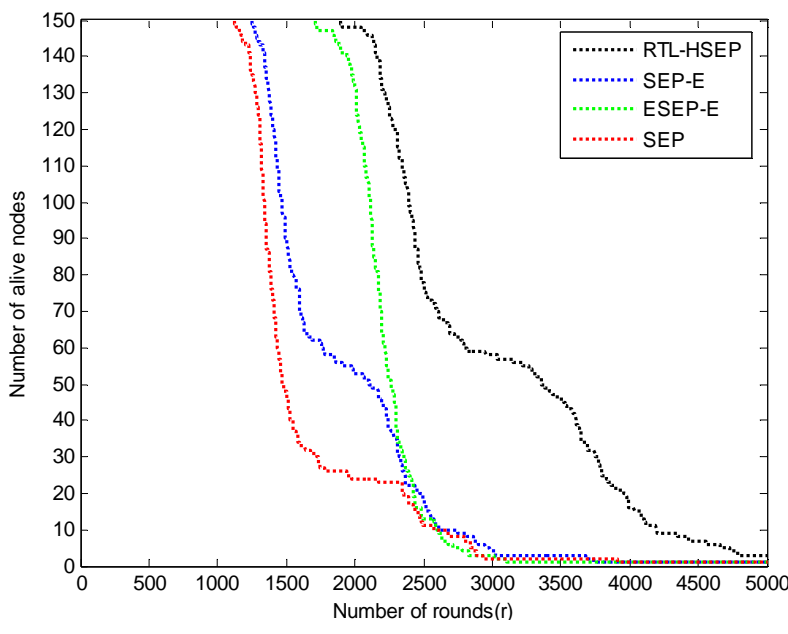

FIGURE II. NUMBER OF NODES ALIVE PER ROUND

Fig. 3 shows the number of rounds for the first node death and half nodes death. RTL-HSEP has longer network life than the other protocols because the half of nodes in RTL-HSEP dies at $2466^{\text {th }}$ round and in SEP, SEP-E and ESEP-E it occurs at $1401^{\text {th }}, 1574^{\text {th }}, 2264^{\text {th }}$ round respectively. Similarly, Stability period of RTL-HSEP is also more than the other protocols because first node in RTL-HSEP dies at $1938^{\text {th }}$ round and in SEP, SEP-E and ESEP-E it happens at $1173^{\text {th }}, 1244^{\text {th }}, 1612^{\text {th }}$ round respectively.

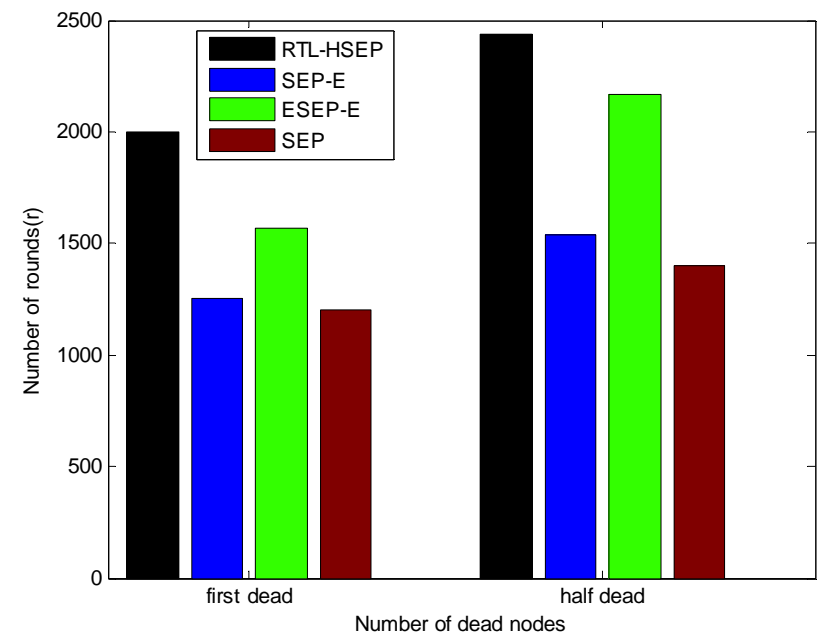

FIGURE III. ROUNDS FOR FIRST, HALF DEATH

Fig. 4 plots the throughput i.e. the data packets received at the base station per round and from the figure it is clear that RTL-HSEP has sent more messages to the base station than the other protocols.

Form all the discussion above, we can see that RTL-HSEP protocol is more suitable for heterogeneous WSNs than the other protocols mentioned here.

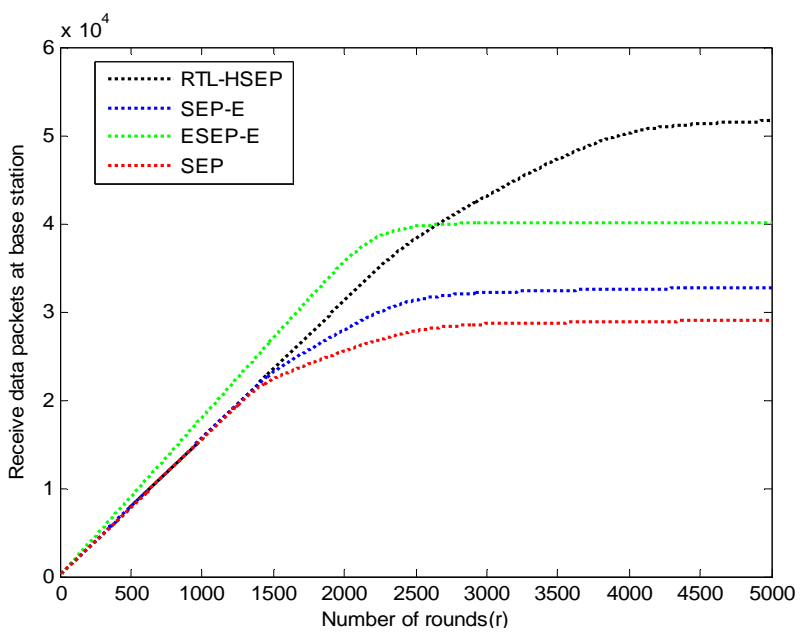

FIGURE IV. TOTAL AMOUNT OF DATA RECEIVED AT BASE STATION

\section{SUMMARY}

One of the main challenges in the design of routing protocols for WSNs is energy efficiency due to the limited energy resources of sensors. In this paper, we propose an improved protocol RTL-HSEP. Reactive routing mechanism is employed to minimize the number of nodes transmitting data to the cluster heads so as to increase the stability period of the network .The simulation results show that the proposed RTL-HSEP protocol is more efficient, and prolong the life cycle of the entire network. However, this paper does not consider the multi-hop between cluster heads and the base station and more works need to be done further on it.

\section{ACKNOWLEDGEMENT}

This research was financially supported by the National Key Technology R\&D Program under No. 2014BAF07B01 and the National Science Foundation under No. 612727539.

\section{REFERENCES}

[1] J. Zheng, Abbas Jamalipour, Wireless Sensor Networks: A Networking Perspective, Wiley-IEEE Press, 2009.

[2] S.K. Singh, M.P. Singh, and D.K. Singh, A survey of Energy-Efficient Hierarchical Cluster-based Routing in Wireless Sensor Networks, International Journal of Advanced Networking and Application , 02 (2010) 570-580

[3] R. Kumar, R. Ranjan Kumar, A. Mundra, Centric query processing in Heterogeneous Wireless Sensor Network, Next Generation Computing Technologies, $20151^{\text {st }}$ International Conference on Dehradun, 2015, pp. 301-306.

[4] P. Garnepudi, T. Damarla, J. Gaddipati, Proactive, reactive and hybrid multicast routing protocols for Wireless Mesh Networks, Computational Intelligence and Computing Research, 2013 IEEE International Conference on Enathi, 2013, pp. 1-7.

[5] P. Kumar S, K. Sharma, K. S. Raju, Swarm and Classical Based Routing Schemes in Wireless Sensor Network, Advanced Computing \& Communication Technologies, 2015 IEEE Fifth International Conference on, 2015, pp. 686-689.

[6] W.R. Heinzelman, A. Chandrakasan, and H. Balakrishnan, Energy-efficient communication protocol for wireless micro sensor networks, Proceedings of the 33rd annual Hawaii international conference on System sciences, 2000, pp. 1-10. 
[7] O. Younis, S. Fahmy, HEED: A hybrid, energy-efficient, distributed clustering approach for ad hoc sensor networks, IEEE Transactions on Mobile Computing, 2004, pp. 660-669.

[8] W.R. Heinzelman, A. Chandrakasan and H. Balakrishnan, An application specific protocol architecture for wireless micro sensor networks, IEEE Transaction on Wireless Communications, 2002, pp. 660-670.

[9] G. Smaragdakis, I. Matta, A. Bestavros, SEP: A stable election protocol for clustered heterogeneous wireless sensor networks, Proceeding of 2nd International Workshop on SANPA, 2004, pp. 164-173.

[10] F.A. Aderohunmu, J.D. Deng, An Enhanced Stabel Election Protocol (SEP) for Clustered Heterogeneous WSN, Dunedin: University of Ontago, 2010, pp. 1177-455X.

[11] S. Singh, E.C.E. Dept, Energy Efficient Election Probability and Cluster Head Selection for Enhancement of SEP-E in Wireless Sensor Networks, Machine Intelligence and Research Advancement (ICMIRA), 2013 IEEE International Conference on Katra, 2013, pp. 82-86. 